\title{
EFECTO DE LA APLICACIÓN DE ONDAS DE ULTRASONIDO DE ALTA POTENCIA (25kHz) SOBRE LAS PROPIEDADES FISICOQUÍMICAS Y MICROBIOLÓGICAS DEL MÚSCULO SEMITENDINOSO LONGISSIMUS DE LA ESPECIE BOVINA.
}

\author{
EFFECT OF THE APPLICATION OF HIGH POWER ULTRASONIC WAVES (25KHZ) ON \\ PHYSICOCHEMICAL AND MICROBIOLOGICAL PROPERTIES OF SEMITENDINOSUS \\ LONGISSIMUS MUSCLE OF BOVINE SPECIES
}

\section{Efecto del ultrasonido sobre las propiedades fisicoquímicas y microbiológicas de un músculo de la especie bovina}

\author{
Effect of Ultrasound on the physicochemical and Microbiological \\ properties Of A Muscle Of Bovine Species
}

\footnotetext{
Ingeniera Química - Instituto Universitario de la Paz

${ }^{2}$ Médico veterinario y Zootecnista-Instituto Universitario de la Paz

${ }^{3}$ Master en ciencia y tecnología de alimentos- Instituto Universitario de la Paz

Recibido: Mayo 5 de 2015 Aceptado: 3 de Agosto de 2015

*Correspondencia del autor: Natalia Terán Acuña, Cra. 38 No. 36 c 47 Barrancabermeja, S., E-mail: natalia.teran@unipaz.edu.co
}

\begin{abstract}
RESUMEN
Se estudió la aplicación de ultrasonido de alta potencia como tecnología no química para mejoramiento de la calidad e inocuidad de la carne, evaluando los efectos de las ondas ultrasónicas sobre las propiedades fisicoquímicas ( $\mathrm{pH}$, cenizas, acidez, actividad acuosa, humedad, capacidad de retención de agua) y microbiológicas (aerobios mesófilos, coliformes totales y fecales) del músculo semitendinoso Longissimus en bovinos machos entre los 4 y 5 años de edad con 12 horas de post-morten. Para el estudio, las muestras fueron sometidas de manera independiente y por triplicado, a tratamientos con ultrasonido a una frecuencia de $25 \mathrm{kHz}$, durante tiempos de exposición de 30, 45 y 60 minutos. Los resultados obtenidos fueron analizados mediante el software comercial SPSS versión 21. Se calculó el análisis de varianza (ANOVA) con diseño completamente al azar y mínima diferencia significativa $\alpha=0,05$. Estos resultado mostraron que tras el tratamiento realizado, propiedades como el $\mathrm{pH}$, la capacidad de retención de agua (CRA), cenizas, acidez y actividad acuosa (aw) no presentan cambios estadísticamente significativos $(\alpha=0,05)$, lo que evidencia que el tratamiento no afecta la calidad de la carne; por otro lado, la humedad del musculo presenta una disminución significativa $(\alpha=0,05)$ debido a la cavitación producida por las ondas de ultrasonido, lo cual genera micro evaporación del agua, mientras que los efectos sobre la carga microbiana incidieron en la disminución de aerobios mesófilos y coliformes totales y fecales sobre el músculo, llegando a una inactivación microbiana con resultados favorables tras periodos de exposición de 60 minutos.
\end{abstract}

Palabras Clave: Capacidad de retención de agua (CRA), Actividad acuosa (aw), Cenizas, pH, Frecuencia, inocuidad, ultrasonido 


\begin{abstract}
Application of high power ultrasound, as a non-chemical technology, to improve the quality and safety of meat was studied, evaluating the effects of ultrasonic waves on physicochemical ( $\mathrm{pH}$, ash, acidity, water activity, moisture, water holding capacity) and microbiological properties (aerobic mesophilic bacteria, total and fecal coliforms) of a semitendinosus muscle Longissimus in male animals between 4 and 5 years old, which had 12 hours post-mortem. For the study, the samples were worked independently and in triplicate whith ultrasound treatment at a frequency of $25 \mathrm{kHz}$, for exposure times of 30,45 and 60 minutes. The results were analyzed using commercial software SPSS version 11.0, by computing analysis of variance (ANOVA) with completely randomized design and significant difference least $\alpha=0.05$. These results showed that after the ultrasound treatment, properties such as $\mathrm{pH}$, water holding capacity (WHC), ashes, acidity and water activity (aw) do not have statistically significant changes $(\alpha=0.05)$ by ANOVA, which evidence that treatment does not affect the quality of meat; other hand, muscle moisture presents a significant decline $(\alpha=0.05)$ due to cavitation caused by the ultrasound waves which generates micro water evaporation, the effects on the microbial load influences a decreasing on aerobic mesophilic, total and fecal coliforms on muscle reaching a microbial inactivation with favorable results after exposure periods of 60 minutes.
\end{abstract}

Keywords: Wather holding capacity, Water activity, ashes, $\mathrm{pH}$, frequency, ultrasound, food safety

\section{INTRODUCCION}

En la actualidad, los consumidores de alimentos se han vuelto cada vez más exigentes en cuanto a las características de aceptabilidad de los productos que adquieren, llevando a los compradores a considerar en los alimentos procesados, la preservación de las características propias del material fresco(1). La aplicación de tratamientos térmicos ha sido la tecnología más utilizada para la estabilización de alimentos debido a su alta capacidad de destrucción de microorganismos e inactivación de enzimas.(2) Sin embargo, su uso implica cambios en las propiedades organolépticas del alimento, además de posibles reducciones en los contenidos y/o la biodisponibilidad de algunos nutrientes(2).

Por lo anterior, durante las últimas décadas se ha generado un creciente interés en la búsqueda y desarrollo de tecnologías que minimicen los efectos negativos de los tratamientos térmicos(3), dando pie a que técnicas como el ultrasonido, se conviertan en alternativas muy promisorias para el procesamiento de alimentos. Las ondas de ultrasonido son características en frecuencias por encima de $20 \mathrm{kHz}$, y se clasifican en ultrasonidos los de alta y baja intensidad o baja frecuencia y alta potencia, $(4,5)$ los primeros no causan alteraciones físicas ni químicas en el material sobre el cual actúan, por lo que son usados en el campo médico, por otro lado, los ultrasonidos de baja frecuencia, "permiten realizar operaciones de estabilización sin incrementar drásticamente la temperatura del sistema y preservan las características sensoriales del producto"(2). Su funcionamiento está basado en la generación de intensos gradientes de presión, temperatura y corte dentro del material sobre el cual actúan y por lo tanto alteran su estructura y reactividad química, lo que posibilita cambios en las propiedades como cristalización de grasas y azucares, inhibición de enzimas, oxidación, congelación y ablandamiento de tejidos.(6) Otro efecto importante está asociado a la capacidad de inhibir el crecimiento de microorganismos debido a que causa daños en su pared celular, por lo cual ofrece ventajas pudiéndose aplicar como una nueva tecnología en la conservación de alimentos.(7)

Uno de los alimentos que representan un consumo importante en la industria alimenticia es la carne fresca, producto altamente perecedero debido a su composición bioquímica y alta actividad acuosa; desde el momento en que es sacrificado el animal, la carne sufre procesos de degradación y contaminación por una diversidad de microorganismos que conducen inevitablemente a su alteración; factores como: la temperatura, el oxígeno atmosférico, las enzimas endógenas, la humedad, la luz, además de condiciones de manejo en el sacrificio del animal, influyen en la vida útil y frescura de la carne, provocando situaciones de contaminación con altas cargas microbianas que pueden llegar a producir problemas de salud pública.(8)

Con la aplicación de técnicas de ultrasonido, se bus- 
ca minimizar los riesgos asociados a la inocuidad del producto cárnico, sin comprometer la calidad del producto6, esto implica la no afectación de propiedades como la capacidad de retención de agua (CRA) y pH, las cuales representa una medida de la calidad del producto y su estado del grado de deterioro(9).

Es de interés en el presente estudio, evaluar el efecto de la aplicación de ondas de alta potencia $(25 \mathrm{kHz})$ sobre las propiedades fisicoquímicas ( $\mathrm{pH}$, cenizas, acidez, actividad acuosa, humedad, capacidad de retención de agua) y microbiológicas (aerobios mesófilos, coliformes totales y fecales) en el músculo semitendinoso Longissimus de la especie bovina, con el fin de determinar la viabilidad de esta técnica en términos de inocuidad y calidad de este producto.

\section{MATERIALES Y MÉTODOS}

El estudio fue realizado, utilizando el músculo semitendinoso Longissimus de cadáveres bovinos machos entre los 4 y 5 años de edad, adquiridos en la planta de beneficio del municipio de Barrancabermeja, Santander, los cuales contaban con 12 horas de post-mortem y condiciones de refrigeración a $7^{\circ} \mathrm{C}$, las muestras inicialmente sufrieron un pre-tratamiento que consistía en retirar todo el material adherente al musculo como la materia grasa. Posteriormente, las muestras fueron cortadas y sometidas a tratamientos con ondas de frecuencia a $25 \mathrm{kHz}$ en un baño de ultrasonido multifrecuencia marca ELMA, TI-H-25 con capacidad para 25 litros (100\% de potencia transmitida al medio). En el estudio se consideraron cuatro muestras por triplicado, una de las cuales corresponde a una muestra sin tratamiento (ST), mientras que las otras tres (T1, T2, T3) se sometieron a la técnica durante periodos de exposición de 30, 45 y 60 minutos respectivamente.

Una vez concluido el tiempo de tratamiento, todas las muestras fueron analizadas para determinar sus propiedades fisicoquímicas y microbiológicas, de acuerdo con los procedimientos establecidos por la Association of Official Analytical Chemists- AOAC, NTC 4516 y 4519, como se indica en la tabla 1.

Tabla 1. Métodos usados para la determinación de propiedades fisicoquímicas

\begin{tabular}{cl}
\hline Propiedad & \multicolumn{1}{c}{ Equipo- Método } \\
\hline \multirow{2}{*}{ pH } & Potenciómetro marca \\
& OAKTON, Modelo: ION 510/ \\
& PhmetroISE. Mobile 827
\end{tabular}

Aerobios mesófilos, coliformes totales y fecales
Titulación, con solución de hidróxido de sodio $(0.1 \mathrm{~N})$ y fenolftaleína como indicador.

Equipo de humedad directo marca Mettler Toledo HB43-S Halogen.

Compresión con papel filtro y determinando diferencias de peso antes y después de la comCRA presión con la ayuda de una balanza analítica marca OHAUS Discovery

Medidor de actividad acuosa directo aw marca Rotronic.

Método oficial AOAC (1996) 920.153, Mufla Terrigeno

Recuento de microorganismos según NTC 4519 y 4516. Medios de cultivo de la casa OXOID

Los resultado obtenidos en cada una de la pruebas, se analizaron mediante el Software comercial (Statistical Products and Service Solution, SPSS versión 21. Inc., Chicago, IL, USA).

Los valores medio y los cuantitativos se compararon mediante análisis de varianza (ANOVA) con diseño completamente al azar, utilizando la mínima diferencia significativa $\alpha=0,05$.

\section{RESULTADOS Y DISCUSIÓN}

La aplicación del tratamiento con ondas de $25 \mathrm{KHz}$ sobre el musculo semitendinoso, genera una caída de $\mathrm{pH}$ proporcional al tiempo de exposición (Tabla 2). El $\mathrm{pH}$ de la muestra ST presenta un valor promedio de 6,38 que disminuye en las muestras tratadas $\mathrm{T} 1, \mathrm{~T} 2 \mathrm{y}$ T3, alcanzando un valor promedio de 5,83 tras 60 minutos de tratamiento. Este comportamiento se atribuye a la disociación de las moléculas de agua producto de la sonólisis, la cual libera iones hidrogeniones al medio ocasionando que disminuya el $\mathrm{pH}(10)$. Estos cambios en el pH se han evidenciado en aplicaciones de ultrasonido para diferentes grupos de alimentos como frutas, verduras y cárnicos(6).

Por otro lado, los procesos post morten en el tejido muscular de la especie bovina, ocasionan que el glucógeno se metabolice en ácido láctico, haciendo que la carne presente disminuciones de $\mathrm{pH}$ hasta un valor final de entre 5,4 a 5,8 después de $24 \mathrm{~h}$ de post-mortem $(11,12)$. Al comparar este valor final de $\mathrm{pH}$ con el 
obtenido tras 60 min de tratamiento con ultrasonido, se pudo evidenciar que la tecnología usada acelera el descenso de esta propiedad, con lo que el $\mathrm{pH}$ final se alcanza en menos tiempo, sin embargo cabe resaltar que los cambios de $\mathrm{pH}$ pueden depender de varios factores como el tiempo de almacenamiento, tipo de músculo y el tiempo de tratamiento(13).

Tabla 2. Cambios de $\mathrm{pH}$ a una frecuencia de $25 \mathrm{kHz}$.

\begin{tabular}{lrrrrcc}
\hline Muestra & E1 & E2 & E3 & X & S & $\mathbf{S}^{\mathbf{2}}$ \\
\hline ST & 6,87 & 6,44 & 5,84 & 6,38 & 0,51733 & 0,268 \\
T1 & 6,20 & 6,40 & 5,81 & 6,14 & 0,30006 & 0,090 \\
T2 & 5,88 & 6,38 & 5,64 & 5,97 & 0,37754 & 0,143 \\
T3 & 5,72 & 6,31 & 5,46 & 5,83 & 0,43555 & 0,190 \\
\hline
\end{tabular}

Nota: E1,E2 y E3: Replica 1,2 y 3 ; X: Promedio; S: Desviación Estándar; $\mathrm{S}^{2}$ : Varianza.

En lo correspondiente al porcentaje de la acidez en el musculo semitendinoso tratado con ultrasonido este presenta ligeros aumentos con el tiempo de exposición (Tabla 3); partiendo de un valor promedio de $0,41 \%$ para las muestras sin tratamiento, hasta $0,57 \%$ para el máximo periodo de exposición, este fenómeno se atribuye a la micro evaporación del agua, producto de la sonólisis, lo que provoca una concentración del ácido láctico presente en el músculo(13).

Tabla 3. Cambios en la acidez expresada en ácido láctico a una frecuencia de $25 \mathrm{kHz}$.

\begin{tabular}{lcccccc}
\hline Muestra & E1 & E2 & E3 & X & S & S $^{2}$ \\
\hline ST & $0,36 \%$ & $0,32 \%$ & $0,54 \%$ & $0,41 \%$ & 0,11719 & 0,014 \\
T1 & $0,45 \%$ & $0,45 \%$ & $0,54 \%$ & $0,48 \%$ & 0,05196 & 0,003 \\
T2 & $0,54 \%$ & $0,50 \%$ & $0,62 \%$ & $0,56 \%$ & 0,06110 & 0,004 \\
T3 & $0,54 \%$ & $0,54 \%$ & $0,63 \%$ & $0,57 \%$ & 0,05196 & 0,003
\end{tabular}

Nata E1,E2y E3: Replica 1,2y 3; X Promedo;

\section{S:Desviación Estandar, S2Variarza}

La actividad acuosa es un parámetro relacionado directamente con la capacidad de conservación del alimento, valores de $a_{w}$ inferiores a 0,65 inhiben totalmente el crecimiento microbiano, mientras que valores superiores favorecen su propagación(14).

Para la carne fresca, el valor de $\mathrm{a}_{\mathrm{w}}$ es de aproximadamente 0.99 , lo que genera un ambiente adecuado para el crecimiento de microorganismos(14). Al someter la muestra al proceso de ultrasonido, se observó que los valores de $\mathrm{a}_{\mathrm{w}}$ disminuyen ligeramente debido a la microevaporación del agua producto de la sonólisis, sin embargo, los cambios presentados no fueron significativos, obteniéndose valores promedio de 0.97 para las muestras ST y T1, y 0.96 para las muestras T2 y T3. (Tabla 4).

Tabla 4. Cambios en la actividad acuosa $\left(a_{w}\right)$ a una frecuencia de $25 \mathrm{kHz}$.

\begin{tabular}{lrrrccc}
\hline Muestra & E1 & $\mathbf{E 2}$ & $\mathbf{E 3}$ & $\mathbf{X}$ & $\mathbf{S}$ & $\mathbf{S}^{\mathbf{2}}$ \\
\hline ST & 0,97 & 0,98 & 0,97 & 0,97 & 0,00577 & 0,000 \\
T1 & 0,96 & 0,98 & 0,97 & 0,97 & 0,01000 & 0,000 \\
T2 & 0,95 & 0,98 & 0,96 & 0,96 & 0,01528 & 0,000 \\
T3 & 0,94 & 0,98 & 0,96 & 0,96 & 0,02000 & 0,000
\end{tabular}

Nota: E1,E2 y E3: Replica 1,2 y 3 ; X: Promedio; S: Desviación Estándar; $\mathrm{S}^{2}$ : Varianza.

De igual manera, el porcentaje de humedad del músculo semitendinoso, presenta una disminución gradual con respecto al tiempo de exposición a ultrasonido, los valores descritos en la tabla 5 evidencian la disminución en un rango de $75,07 \%$ a $73,6 \%$ para la muestra sin tratamiento y muestra con mayor exposición respectivamente, este comportamiento se explica a través de la microevaporación del agua causada por el fenómeno de cavitación(13), este efecto aunque es estadísticamente significativo no representa una alteraciones en las características de calidad, ni en el proceso de conservación de la carne.

Tabla 5. Cambios en la Humedad del músculo semitendinoso Longissimus.

\begin{tabular}{|c|c|c|c|c|c|c|}
\hline Mestra & E & E & E & $X$ & $S$ & $s^{2}$ \\
\hline ST & 74,56 & $74,72^{\circ}$ & 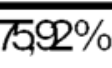 & $75 \% \% 0$ & 274333 & \\
\hline T1 & 73,83 & $73,79 \%$ & $74,66 \%$ & $74,13 \% 0$ & 2548006 & \\
\hline T2 & 73,72 & 7365 & $7379 \%$ & $7372 \% 0$ & 20000 & \\
\hline T3 & 7369 & $7356 \%$ & $7372 \%$ & $7366 \% 0$ & 208565 & Q Q \\
\hline
\end{tabular}

El análisis de las muestras de músculo semitendinoso evidencia un incremento en los valores de agua retenidos por el papel filtro durante el proceso de compresión (tabla 6), se identificó un valor promedio de $2,02 \%$ para la muestra ST, el cual aumenta para las experiencias realizadas con periodos de exposición de 15,30 y 60 minutos, alcanzando para esta ultima un valor promedio máximo $6,17 \%$. Esta variación denota una disminución en la CRA, propiedad relacionada con la textura, terneza y color de la carne(11), descensos de esta propiedad pueden provocar una pérdida 
de peso considerable y una textura reseca del producto(2).

Los cambios en la CRA afectan al agua que se denomina "inmovilizada" y no tienen ninguna relación con el "agua de constitución" (fuertemente ligada a grupos específicos de la molécula o ubicada en regiones intersticiales) ni tampoco con el "agua de interfase"(15), se infiere entonces que el incremento en el porcentaje de agua retirada por compresión, se debe al fenómeno de cavitación producto de la aplicación de ultrasonido, ya que este genera gradientes de temperatura y compresión y descompresión alternativa del material, produciendo microcanales en las interfaces del material lo que permite mayor movilidad del agua(16).

Tabla 6. Cambios en la capacidad de retención de agua (CRA).

\begin{tabular}{|c|c|c|c|c|c|c|}
\hline dra & $\theta$ & E2 & B & $\bar{X}$ & $S$ & $\$$ \\
\hline & $1,65 \%$ & $1, \pi 0 \%$ & $330 \%$ & $20 \%$ & 1,15794 & 1,34 \\
\hline & $215 \%$ & २२\% & $610 \%$ & $350 \%$ & 25202 & 5073 \\
\hline & $290 \%$ & $245 \%$ & $7,10 \%$ & $4,15 \%$ & 256466 & 658 \\
\hline & $345 \%$ & ५ऽ\% & $98 \%$ & $67 \%$ & $3 य \nabla 4$ & 10711 \\
\hline
\end{tabular}

NtaE1,E2yE3 Replica1,2y3; XFoneda

\section{SLesiadónEstand; S2Vaiaza}

Los resultados obtenidos con respecto a la disminución de la CRA son coherentes con estudios realizados por Jayasooriya et al(13), donde se evidencia que descensos en el $\mathrm{pH}$ ocasionan disminución de la capacidad de retención, esto se debe a que a medida que el $\mathrm{pH}$ se acerca al punto isoeléctrico de las proteínas que conforman el músculo, se presenta una cierta difusión y redistribución iónica que da como resultado el debilitamiento de las fuerzas electrostáticas(11), y por consiguiente las moléculas de agua que se encontraban retenidas a la proteína son liberadas.

Los valores de porcentaje de cenizas obtenidos para el análisis del musculo estuvieron comprendidos dentro de los rangos habituales para la carne(11), tanto para la muestra sin tratamiento como para las sometidas a sonólisis. En la tabla 7 se pueden observar los cambios presentados en el porcentaje de cenizas, los cuales comprenden variaciones entre $1,35 \%$ a $1,10 \%$, evidenciando que el tratamiento aplicado no genera alteraciones significativas en esta variable; al comparar estos resultados con los obtenidos por Martínez y Astiasarán(17) con el uso de métodos de vapor, se evidencia que la técnica de ultrasonido presenta menor afectación en el contenido de cenizas que la técnica de vapor, ya que esta última genera pérdidas en vitaminas y minerales debido a la volatilización.

Tabla 7. Cambios en el $\%$ de cenizas a una frecuencia de $25 \mathrm{kHz}$.

\begin{tabular}{lcccccc}
\hline Muestra & E1 & E2 & E3 & X & S & $\mathbf{S}^{2}$ \\
\hline ST & $1,35 \%$ & $0,96 \%$ & $1,57 \%$ & $1,29 \%$ & 0,30892 & 0,095 \\
T1 & $1,23 \%$ & $0,96 \%$ & $1,40 \%$ & $1,20 \%$ & 0,22189 & 0,049 \\
T2 & $1,28 \%$ & $0,96 \%$ & $1,30 \%$ & $1,18 \%$ & 0,19079 & 0,036 \\
T3 & $1,10 \%$ & $1,20 \%$ & $1,20 \%$ & $1,17 \%$ & 0,05774 & 0,003 \\
\hline
\end{tabular}

Nbta: E1,E2 y E3: Replica 1,2 y 3; X Promedio;

\section{S:Desviación Estandar, S2:Variarza.}

En la tabla 8, se muestran los resultados de la carga de microorganismos mesófilos presentes en el músculo semitendinoso, para las muestras analizadas; aunque la carga inicial resultó ser baja, al someter la muestra a ultrasonido, se logró una reducción estadísticamente significativa $(\alpha=0,05)$, demostrando el efecto directo del tiempo de exposición a ultrasonido sobre la carga bacteriana de la carne.

Tabla 8. Resultados del recuento total de microorganismos aerobios mesófilos a una frecuencia de $25 \mathrm{kHz}$.

\begin{tabular}{|c|c|c|c|c|c|c|}
\hline Mestra & EI & E & $E B$ & $\mathrm{X}$ & $S$ & $s^{2}$ \\
\hline ST & $1,96+\infty$ & 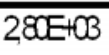 & $2 / \omega, \omega$ & $22 \pi E+C B$ & $4,75 E+C 2$ & $222 E+05$ \\
\hline 1 & $1,3 \mathrm{BE}+C B$ & $y \in+B$ & $1, \pi E+C B$ & 1, GE+CB & 306E+OD & $935+04$ \\
\hline 2 & $8 G E+C$ & 1,35E+C3 & $7, \pi E+C R$ & $97 \pi E+C R$ & 284E+CD & $802 E+04$ \\
\hline & $7, Q \mathrm{EE}+Q 2$ & $855 E+C 2$ & $4,8 E+C 2$ & 7,OCE'CQ & $1,9 \pi+C R$ & 3\&E+O4 \\
\hline
\end{tabular}

Nta E1,E2yE3 Redica1,2y3; XFronedq

SLesvicán Estanda, S2Vaiaza

La reducción obtenida en la carga de mesófilos, están en concordancia con estudios realizados por Álvarez(18), y se atribuye a la inactivación de la carga microbiana por el efecto letal del ultrasonido de alta potencia y al fenómeno de cavitación, el cual genera altas presiones y altas temperaturas que conduce a la formación de radicales libres los cuales son muy reactivos y pueden producir oxidación de los fosfolípidos que componen las membranas celulares de los microorganismos, y en consecuencia la posterior inactivación de las células bacterianas.

Por su parte, Mason(19) determina que la disminución de los microorganismos mesófilos en el musculo 
semitendinoso se relaciona con los daños producidos por el ultrasonido en la pared celular de los mismos y que como resultado ocasiona la destrucción de la célula viva.

Un comportamiento similar se observó en lo correspondiente a microorganismos coliformes totales $\mathrm{y}$ fecales como se presenta en la tabla 9 y 10 respectivamente, donde se evidencia que el tratamiento de ultrasonido ocasiona una reducción de la carga microbiana a medida se aumentan los tiempos de exposición al tratamiento. Estos resultados coinciden con el estudio realizado por Jayasooriya et al(13), donde se encontró que la aplicación de ultrasonido tiene un efecto germicida sobre la flora microbiana presente en la carne bovina, el cual depende de la intensidad del tratamiento, tiempo y temperatura durante el tratamiento.

Tabla 10. Resultados del recuento total de microorganismos Coliformes fecales a una frecuencia de $25 \mathrm{kHz}$.

\begin{tabular}{|c|c|c|c|c|c|c|}
\hline Muestra & E1 & $\mathrm{E} 2$ & $E 3$ & $\bar{x}$ & $\mathrm{~S}$ & $\mathrm{~S}^{2}$ \\
\hline ST & 1,10E+CB & $24 C E+\infty 3$ & 2,40E'@B & 1,97E+CB & 7,51E+CR & $5,65+05$ \\
\hline T1 & 4, שEE+CR & 1,10E+CB & 1,10E+CB & $8,8 / E+C R$ & $3,70 E+C R$ & 1,3/E+05 \\
\hline T2 & $2,40 E+C R$ & $1,50 E+01$ & $9,30 \mathrm{E}+01$ & 1,1EE+CR & 1,14E+CR & $1,31 \mathrm{E}+04$ \\
\hline $\mathrm{T3}$ & $230 E+01$ & $1,10 E+01$ & 2,10E+O1 & 1,83E+01 & $6,43 E+\infty$ & 4, 13E+01 \\
\hline
\end{tabular}

\section{CONCLUSIONES}

En la investigación, se evidencio el efecto de la aplicación de ondas de ultrasonido de alta potencia $(25 \mathrm{kHz})$ sobre las propiedades fisicoquímicas y microbiológicas del musculo semitendinoso longissimus de la especie bovina.

En el estudio se lograron identificar descensos en el
$\mathrm{pH}$, proporcional al tiempo de exposición, alcanzando reducciones desde un valor inicial de 6,38 hasta 5,83, debido a la producción de ácido láctico, así mismo como incrementos en la acidez, por lo que se alcanza a través de la sonólisis un posible proceso de maduración acelerado, reduciendo el tiempo normal de maduración de carne en refrigeración de 3-4 días a una hora.

También cabe señalar que las ondas ultrasónicas causaron reducción de humedad, actividad acuosa y la CRA, cambios atribuidos al fenómeno de cavitación que genera microevaporación del agua, sin embargo, aunque estos resultados no garantizan mejoras en la conservación del alimento, tampoco llegan a deteriorar las características bromatológicas del mismo.

Finalmente se determinó que el efecto del ultrasonido ocasiona una reducción en la carga microbiana (mesófilos aerobios, y coliformes totales y fecales) presente en el musculo semitendinoso, la cual se relaciona con el daño que produce el ultrasonido en la pared celular de los microorganismos y a su vez la destrucción de la célula viva. Esta reducción se presenta en la medida en que se aumenta el tiempo de exposición a una frecuencia de $25 \mathrm{kHz}$ obteniendo un efecto casi letal en el tiempo de $60 \mathrm{~min}$. Por lo tanto cabe resaltar que la aplicación del ultrasonido tiene un alto poder destructivo de microorganismos.

Con base en los resultados obtenidos, se recomienda realizar fututas investigaciones empleando la combinación de ultra sonido con otras técnicas de conservación de alimentos, con el fin de ofrecer nuevas alternativas a la industria alimentaria, asimismo, evaluar los efectos de los tratamientos de ultrasonido sobre las propiedades como textura, características microscópicas y composición en la cantidad de proteínas. 


\section{BIBLIOGRAFÍA}

1. Azuola R, Vargas P. Extracción de sustancias asistidas por Ultrasonido. Tecnología en marcha. 2007; p. 30-40.

2. González G. Aplicación de ultrasonidos para la estabilización de sistemas alimentarios. En: II Congreso de Desarrollo Agroindustrial. Instituto Universitario de la Paz UNIPAZ. Barrancabermeja. 2005 p. $1-5$

3. López AG, Figueroa J, Mendoza A, Martínez H, Ramírez B, Morales E, García I. The ultrasound technique for predicting wheat quality on single kernels. Revista Fitotecnia Mexicana. 2006; p.163-169.

4. Javanaud C. Applications of ultrasound to food systems. Ultrasonic's. Vol. 26. 1998; p. 117-123.

5. Suslick, KS. Ultrasound: Chemical, biological and physical effects. VCH Publishers.1998: p 123-163.

6. Pingret D, Fabiano-Tixier AS, Chemat. F. Degradation during application of ultrasound in food processing: A review. Food control 2013; p 593-606

7. Turantaşa F, Kılıçc GB, Kılıçc B. Ultrasound in the meat industry; general applications and decontamination efficiency. International Journal of Food Microbiology 2014; disponible en: 10.1016/j.ijfoodmicro.2014.12.026

8. ZHOU GH, XU XL, LIU Y. Preservation technologies for fresh meat. Meat Science 86. 2010; p. 119 $-128$

9. Ocampo Ibáñez ID, Bermúdez F, Díaz H. Efecto del tiempo de almacenamiento, el tipo de músculo y el genotipo del animal sobre las pérdidas por goteo en carne cruda de cerdo. Acta Agronómica. Ed 3. vol. 58 p. 180-188, dic. 2009. ISSN 2323-0118. Disponible en: $<$ http://www.revistas.unal.edu.co/index. php/acta_agronomica/article/view/11514/12168>. Fecha de acceso: 15 feb. 2015

10. KODA S, KENJI Y, HIROYASU Y. Effect of power ultrasound on $\mathrm{pH}$ change in water saturated with air, oxygen, nitrogen, argon and mixtures. Kagaku Kogaku Ronbunshu. Vol. 25 1999; p. 290-293

11. Huff-Lonergan E, Lonergan SM. Mechanisms of water-holding capacity of meat: The role of postmortem biochemical and structural changes. Meat Science 2005; p. 194-204

12. Koniecko E. Hand book for neat chemists, avery. Nueva Jersey: Publishing Group Inc. 1999. p 325 $-329$

13. Jayasooriya SD, Torley PJ, D'arcy BR, Bhandari BR. Effect of high power ultrasound waves on properties of meat: A review. International Journal of Food Properties 2004; p. 301-319

14. Cheftel JC, Cheftel H. Introducción a la bioquímica y tecnología de los Alimentos. Volumen I. Zaragoza: Acribia: 1992.

15. Departamento de producción animal. REVISIÓN BIBLIOGRÁFICA: Capacidad de retención de agua. Disponible en: http://www.uco.es/organiza/departamentos/prod-animal/economia/aula/img/pictorex/07_09_40_3_REVCRA.pdf

16. Ozuna C, Puig A, García-Pérez JV, Mulet A, Cárcel JA. Influence of high intensity ultrasound application on mass transport, microstructure and textural properties of pork meat (Longissimus dorsi) brined at different $\mathrm{NaCl}$ concentrations. Journal of Food Engineering 2013; p. 84-93

17. Astiasarán I, Martínez JA. Alimentos. composición y propiedades. Segunda reimpresión. México, D.F.: McGraw-Hill interamericana: 2003; p. 17.

18. Álvarez I, Manas P, PAGAN R. Microbial inactivation by ultrasound food. En: Technology University of Zaragoza. Zaragoza 2005; p. 1-6.

19. MASON TJ. The uses of ultrasound in food technology. En: Ultrasonic's Sonochemistry. Vol. 3 1996; p. $253-260$. 